\title{
Measuring Attitudes towards 'Green' Purchases: A Study of University Students in Kyrgyzstan
}

\author{
Ibrahim Keles*, Turdukan Bekimbetova \\ Department of Management, International Ataturk Alatoo University, 720048, Bishkek, Kyrgyzstan \\ *Corresponding Author: ibrahim.keles@iaau.edu.kg
}

Copyright $(2013$ Horizon Research Publishing All rights reserved.

\begin{abstract}
The Theory of Planned Behavior, based on the value - attitude - behavior hierarchy, is a reliable instrument for measuring green purchasing behavior in different cultural settings. This study examines the application of a sub-section of Theory of Planned Behavior (TPB), namely the measurement of Attitudes Towards Green Purchases (AGP) of university students in Kyrgyzstan. Among American and Chinese consumers, Ecological Affect (EA) has a greater impact on their attitude formation than does Ecological Knowledge (EK). To see if this is also true for Kyrgyz consumers, university students $(n=230)$ were surveyed to measure their ecological affect, ecological knowledge and attitudes towards green purchases. And also try to analyses whether gender plays a role in AGP. The results of the study did not prove any of the hypotheses. More studies must be done the understand Kyrgyz consumers.
\end{abstract}

Keywords Theory of Planned Behavior, Kyrgyzstan, Green Purchase

\section{Introduction}

Sustainable energy, organic food, green technology and green products are buzzwords in popular culture, consumer publications and business school course outlines today. Both government and market researchers alike have been studying the reasons consumers purchase green products or make other environmentally friendly decisions. Providing for the environmental concerns of customers is a win-win strategy for the planet and the firm. Green consumers have been shown to be willing to pay a higher price for environmentally friendly products, which is a huge opportunity for companies as well as governments looking to make eco-friendly policy changes $[1,2]$. The knowledge of the existence of these green buyers is good news for the interested parties, but the task of learning 'who they are' becomes greatly important. Even more important might be finding out how consumers can be transformed into green purchasers. When considering all of the factors that make encouraging a desired behavior in consumers difficult, including limited financial resources, understanding what influences consumers' decisions to purchase green products would be extremely valuable to policy makers and marketers alike.

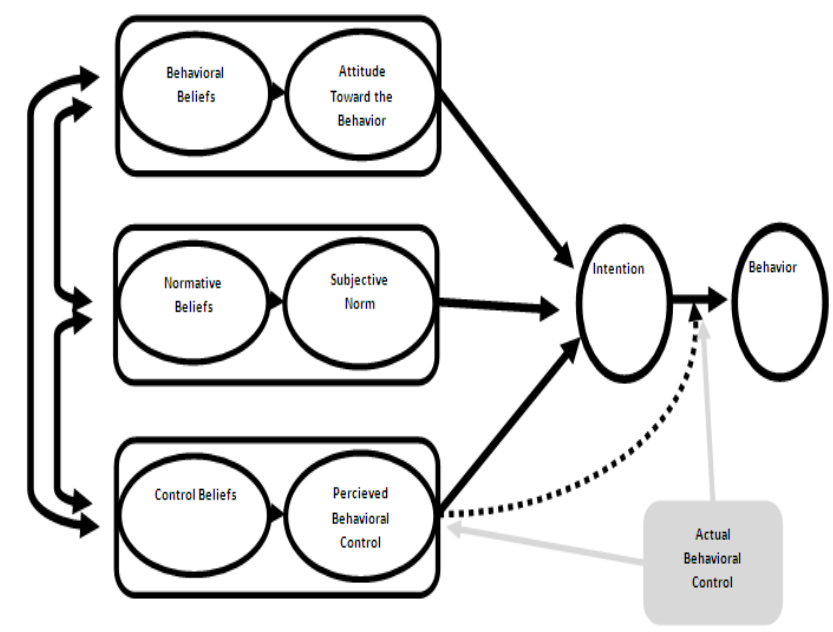

Source [3]

Figure 1. Graphical Representation of the Theory of Planned Behaviour

The intention of the above mentioned study was to seek a better understanding of the various factors that determine the performance of environmentally responsible acts. Studies have suggested that because purchasing green products is a decision, an individual's attitude greatly affects his willingness to perform that action. Two key determinants of attitudes are affect and cognition, or knowledge [4]. In the present situation these are viewed in an ecological way. Ecological Affect (EA) refers to a person viewing him or herself in a particular situation or being influenced, personally, by particular circumstances. Ecological Knowledge (EK) refers to a person's understanding of the factual evidence surrounding environmental issues.

Although TPB considers an affective construct in consumers' formulation of attitudes, it does not include a cognitive construct. Another study [5], focusing on the formulations of attitudes, adds cognitive, affective and cultural constructs to the underlying value - attitude behavior hierarchy existing within the TPB to measure green 
purchase behavior in Chinese consumers. This hybrid model postulates that there are direct causal links between two cultural value dimensions (man-nature orientation and collectivism) and attitudes towards green purchases. It further showed that knowledge and affect also influence individuals' attitudes. The model is loyal to the other aspects of TPB; that an individual's behavior is determined by her behavioral intention to perform that behavior and behavioral intention is a function of the individual's attitude toward the behavior. Figure 2 depicts the model graphically.

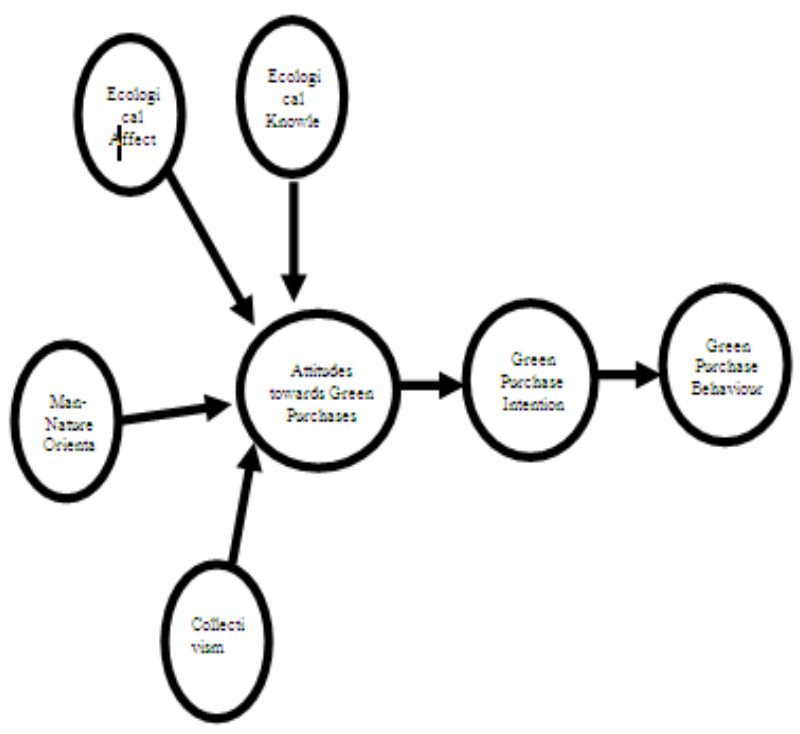

Figure 2. Graphical Representation of the Hybrid Model

Previous studies have found that ecological affect and ecological knowledge influence consumers attitudes towards green products $[1,6,7]$. Chang shows that ecological affect has a greater influence than ecological knowledge in Chinese consumers [4]. This study intends to test the same criteria on university students in Kyrgyzstan to see if the findings hold true. The sample in Chang had a high school graduate education level, on average, while the sample in this study will be high school graduates, at minimum [4]. There is a possibility that a higher education level may cause ecological knowledge to influence attitudes towards green products in a greater way than in previous studies. The results of this study could help influence marketing strategy and government policy in regards to promoting positive attitudes towards environmental decisions in consumers.

\section{Research Question and Hypothesis}

The research questions addressed in the present study are; which factors determine consumer attitudes towards green purchases? As stated above, there are many factors, but the focus in this paper will be on cognitive and affective influences. Therefore the second question of this study is; between cognitive and affective factors, which has a greater influence on AGP? Although an underlying area of interest for this study is whether or not higher education levels can cause EK to have an elevated influence on AGP, this study aims to maintain the results of the previous literature in the formulation of its hypotheses. In order to measure the strength of the influence of ecological affect and knowledge, this study formulates three hypotheses. The first addresses ecological affect and postulates that students with high ecological affect scores will show a statistically significant difference on an attitude towards green purchases scale than students with low ecological affect scores. Based on the results found in the previous literature, the second hypothesis postulates that a significant difference will not be shown in reference to AGP scores between students with high ecological knowledge and those with low ecological knowledge. Finally, if the null hypothesis can be rejected in both hypothesis one and hypothesis two, then hypothesis three, stated; ecological affect will exert a stronger influence on attitudes towards green purchases than ecological knowledge, will have been proven. A comparison of hypotheses one and two will determine the outcome of hypothesis three.

\section{Methodology}

The model used to measure the influence of ecological effect and ecological knowledge on green purchase attitudes is represented in figure-3.This model is a simplified version of the model used in Chang and was meant to measure the cognitive and affective structure of attitudes and purposely did not attempt to measure the influence of culture on green purchase attitudes nor did it look at the affect of green purchase attitudes on green purchase intention or its affect on green purchase behavior, which were measured in the previously mentioned study $[4,8]$.

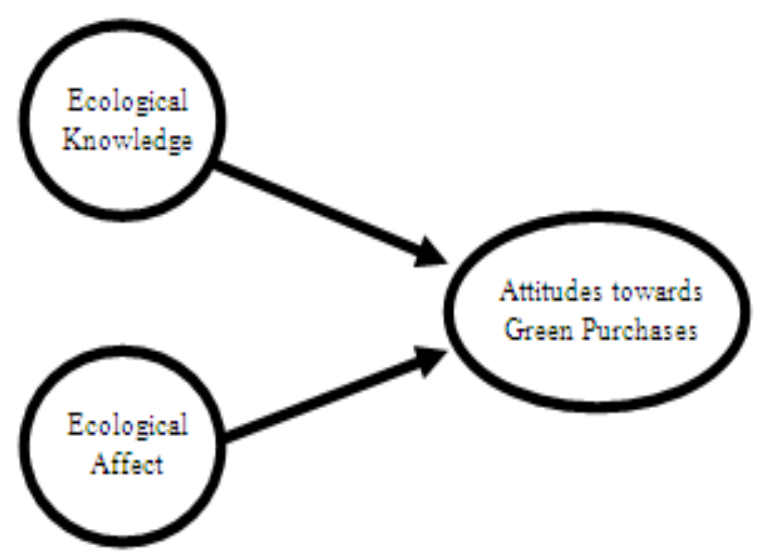

Figure 3. T he Proposed Model of the Present Study

\subsection{The Survey}

To measure the above model, a four-part survey was used. In the demographic measurement section of the survey participants were asked to identify their gender. All participants were undergraduate university students so that they are in the similar age group and have similar education 
levels. The demographic section was followed by an explanation of the terms used in the survey, mainly the working definition for green products. The definition comes from Chang \& Lau and is stated on the survey as; "products that either through their manufacturing or usage have a reduced negative impact on the environment when compared to their traditional counterparts."[5] The second, third and fourth sections recorded AGP, EK and EA respectively (explained in more detail below). The survey was distributed physically, with on-site completion and retention in International Ataturk Alatoo University of Bishkek, Kyrgyzstan. An evaluation of the survey concluded that the errors had been eliminated and resulted in 230 usable responses.

\subsection{Measuring Attitudes towards Green Products}

Participants were given three statements coded by a 7-point semantic differential scale ( 1 to 7 ) to determine their attitudes towards green purchases. These statements are included as Appendix A. The mean scores of these three statements were used to measure the significance of the high and low ecological affect and knowledge groups.

\subsection{Measuring Ecological Affect and Ecological Knowledge}

Once attitude scores were measured then ecological affect (EA) and ecological knowledge (EK) items were measured using an adapted ecological scale from the one used in Chang to assess respondents affect and knowledge relating to general ecological issues [4]. The ecological affect scale was compromised of five statements on a 5-point Likert scale, with $1=$ strongly disagree and $5=$ strongly agree. The fifth question on the Ecological Affect scale had reverse weighting, meaning that marking 1 was calculated as having 5 points and marking 5 was assigned 1 point. The mean of the points were recorded as the score on the EA scale (1 to 5). The ecological knowledge instrument contained five five-category multiple-choice questions. Each correct answer resulted in one point and the sum of the correct answers comprised the score on the EK scale ( 0 to 5$)$. The final ecological knowledge - ecological affects scale used in this study are found in appendix [4]. Chang notes that this method was often used to measure cognitive and affective constructs in previous studies and is one of the most widely used in ecological studies.

\subsection{Measuring the Influence of EA and EK on AGP}

Although the questions and statements used in the EA and EK scales contain the same information as used in Chang, the statistical measurement of EA and EK was different in this study [4]. The scores on the 5-point Ecological Affect Likert Scale were used to divided the participants into two groups, those who had high ecological affect (an mean score of 4 or 5 on the scale, based on a median split of the two groups) and those who had low ecological affect (scores of 1 to 3 on the scale). This division method resulted in the high EA group having 71 participants while the low EA group had 159. A similar division was performed on the participants' scores on the Ecological Knowledge questionnaire. It was divided based on a standard pass-fail evaluation of the five multiple-choice questions. Those participants who answered three or more of the questions correctly were included in the 'high EK' group and those who answered less than three of the questions correctly were included in the 'low EK' group. The numbers in the high EK and low EK groups were 42 and 188, respectively. A two-tailed t-Test with two samples assuming equal variances was used to compare the participants' mean scores on the AGP scale in the high Ecological Affect group and low Ecological Affect group. The same comparison was performed on the high and low Ecological Knowledge groups, and genders.

\subsection{Participants}

A convenience sample was used in this study and the survey was administered in International Ataturk Alatoo University of Bishkek, Kyrgyzstan. As stated above, the survey resulted in 230 responses. 129 participants were male and the remaining 101 were female. Because of having a homogenous group no questions were asked about their education level or ages. A sample copy of the survey administered to the participants is found in appendix part.

\section{Results}

\subsection{Descriptive Statistics}

To provide an overview of the constructs under investigation, Table 1 was developed to display the relevant descriptive statistics for AGP, EA (including high and low groups) and EK (including high and low groups). It should be noted that the descriptive statistics provide some initial support for the hypotheses formulated in this study. As noted in the table, the mean score for the high EA group is significantly higher than the mean score for the low EA group. But it is opposite in between the mean scores of the high EK and low EK groups. And according to gender classification, female respondents have a little higher mean scores comparing to males.

\subsection{Ecological Affect}

To test the first hypothesis, a comparison of high EA and low EA in relation to AGP means, a two-tailed t-Test of two samples assuming equal variances was performed. The results did not support the hypothesis that high EA differs significantly from low EA on an AGP scale. The null hypothesis cannot be accepted with the $\mathrm{P}$ value of $\mathrm{T}$ value $\mathrm{P}(\mathrm{T}<=\mathrm{t})(0.098)>0.05$. 
Table 1. Descriptive Statistics of the Major Constructs under Investigation

\begin{tabular}{|c|c|c|c|}
\hline Major & Number of & Mean & Standard \\
\hline Construct & Responses & Value & Deviation \\
\hline AGP* & 230 & 5.03 & 1.36 \\
\hline EA** $^{*}$ & 230 & 3.50 & 0.68 \\
\hline High EA* & 71 & 5.36 & 1.28 \\
\hline Low EA* & 159 & 4.89 & 1.38 \\
\hline EK** & 230 & 1.00 & 1.64 \\
\hline High EK* & 42 & 4.63 & 1.58 \\
\hline Low EK* & 188 & 5.13 & 1.29 \\
\hline Gender Male & 129 & 4.99 & 1.35 \\
\hline Female & 101 & 5.09 & 1.38 \\
\hline Notes: & & & \\
\hline
\end{tabular}

* Indicates that the score is derived from the 7 point AGP scale.

** Indicates that the score is derived from the 5 point EA/EK scale.

\subsection{Ecological Knowledge}

To test the second hypothesis, comparing the means of high EK and low EK in relation to AGP, a two-tailed t-Test of two samples assuming equal variances was performed. The results did not support the hypothesis that high EK has significant difference from low EK on an AGP scale. The null hypothesis cannot be accepted with the $\mathrm{P}$ value of $\mathrm{T}$ value $\mathrm{P}(\mathrm{T}<=\mathrm{t})(0.196)>0.05$.

\subsection{Gender}

To test the third hypothesis, comparing the means of male and female respondents in relation to AGP, a two-tailed t-Test of two samples assuming equal variances was performed. The results did not support the hypothesis that male respondents have significant difference from female respondents on an AGP scale. The null hypothesis cannot be accepted with the $\mathrm{P}$ value of $\mathrm{T}$ value $\mathrm{P}(\mathrm{T}<=\mathrm{t})(0.813)>0.05$.

\section{Discussion \& Conclusion}

Previous research and studies show that the Theory of Planned Behavior and other value - attitude - behavior hierarchy based models can provide a satisfactory measurement of green purchase behavior. There has also been evidence to show that the inclusion of cognitive and affective aspects of values explains some of the factors that influence attitude formation. Although the TPB model and its hybrids had been tested on samples from different countries, but it had not been used to measure the attitudes of university students in Kyrgyzstan. The current study adds to the knowledge base by the findings of the previous researches with participants from Kyrgyzstan but not supporting. There are several limitations to the current study that will be addressed below.

Although this study attempted to emulate previous literature and models and uncovered similar results, many limitations exist. Obviously when attempting to apply the models used in previous studies, yet not collecting the same information or applying the same statistics to the results causes a major error in experimental design. Due to the nature of this study and its criteria the departure from previous work is acceptable, however further research should investigate the application of the model used in Chang in Kyrgyzstan [4]. Applying the model to two distinct cultures might highlight other values that lead to behavior or the strength of one variable over another in two different populations. The sample size in this study also posed a limitation. Where the noted studies had sample sizes of over 300 participants in each group, the 230 participants in this study is limiting.

And also, this study cannot prove the relation among these three things with AGP. Perhaps, by expanding the study, adding some more demographic factors such as age, education level, earning amounts may show which factors determine purchases towards green purchases for Kyrgyz consumers.

\section{REFERENCES}

[1] Laroche, M., Bergeron, J., Barbaro-Forleo, G. (2001). Targeting consumers who are willing to pay more for environmentally friendly products. Journal of Consumer Marketing, 18 (6), 503-520.

[2] Peattie, K. (2001). Golden goose or wild goose? The hunt for the green consumer. Business Strategy and the Environment, 10 (4), 187-199.

[3] Ajzen, I. (2008). Consumer attitudes and behavior. In C. P. Haugtvedt, P. M. Herr \& F. R. Cardes (Eds.), Handbook of Consumer Psychology (pp. 525- 548). New York: Lawrence Erlbaum Associates.

[4] Chang, R.Y.K. (2001). Determinants of Chinese consumers' green purchase behavior. Psychology \& Marketing, 18 (4), 389-418.

[5] Chang, R.Y.K., Lau, L.B.Y. (2001). Explaining green purchase behavior: A cross-cultural study. Journal of International Consumer Marketing, 14 (2/3), 9-40.

[6] Saphores, J. M., Nixon, H., Ogunseitan, O.A., Shapiro, A.A. (2007). California households' willingness to pay for 'green' electronics. Journal of Environmental Planning and Management, 50 (1), 113 - 133.

[7] Tanner, C., Sybille, W.K. (2003). Promoting sustainable consumption: Determinants of green purchase by Swiss consumers. Psychology \& Marketing, 20 (10), 883-902.

[8] Beyer K. M. (200x). Measuring Attitudes Towards 'Green' Purchases: A Study of University Students in Taiwan, National Chiao Tung University 